\title{
Scattering Calculations with Wavelets
}

\author{
B. M. Kessler, G. L. Payne, W. N. Polyzou* \\ The University of Iowa, Iowa City, IA 52242, USA
}

October 24, 2018

\begin{abstract}
We show that the use of wavelet bases for solving the momentum-space scattering integral equation leads to sparse matrices which can simplify the solution. Wavelet bases are applied to calculate the K-matrix for nucleon-nucleon scattering with the s-wave Malfliet-Tjon V potential. We introduce a new method, which uses special properties of the wavelets, for evaluating the singular part of the integral. Analysis of this test problem indicates that a significant reduction in computational size can be achieved for realistic few-body scattering problems.
\end{abstract}

\section{Introduction}

In this paper we show that bases of compactly supported wavelets can lead to a significant reduction in the size of the matrices used to solve few-body momentum-space scattering integral equations. When the kernel of the integral equation is expanded in a wavelet basis, the resulting matrix can be expressed as the sum of a sparse matrix and a matrix with small norm. Ignoring the small matrix leads to an accurate approximate solution.

Our interest in wavelet bases is motivated by the successful application of wavelets to data compression problems. The coefficients of a wavelet basis provide a compact representation for storing information that has smooth structures on different scales. Wavelets are used for data compression in the FBI fingerprint archive [2] and JPEG files [3]. The property that makes wavelets useful for data compression suggests that they may also lead to sparse matrix representations of the kernels of the momentum-space scattering integral equations. Conventional methods lead to dense matrix representations of these equations; the computation of the solution of these equations for realistic models requires solving large systems of linear equations.

While the use of wavelet bases lead to sparse matrices in both momentum space and configuration space, the resulting reduction in the size of the equations

*This work was supported in part by the Department of Energy, Nuclear Physics Division, under contract DE-FG02-86ER40286 
in momentum space is significant, because the momentum-space kernels are represented by full matrices. These advantages are important for relativistic scattering problems, which are naturally formulated in momentum space.

There are many different types of wavelets that are used in specific applications. In this paper we use the compactly supported wavelets introduced by Daubechies [4] in 1988 to evaluate the K-matrix for two spinless nucleons interacting with an s-wave Malfliet-Tjon V potential [1]. Nucleon-nucleon scattering with the Malfliet-Tjon $\mathrm{V}$ potential provides a good test of numerical methods because the on-shell energy and strength of the attractive and repulsive parts of the potential lead to a problem with three different momentum scales.

The Daubechies wavelets were the first non-trivial set of orthonormal wavelets of compact support discovered, and they have a number of properties that make them useful for numerical applications. However, the Daubechies wavelet basis functions involve a significant amount of structure that can make it difficult to appreciate some of these advantages. Some of the properties that we have found useful are briefly discussed below. They will be explained in more detail in section two.

In applications, there are two bases, called the wavelet basis and the scaling basis. They are related by an orthogonal transformation, called the wavelet transform. This orthogonal transformation can be implemented efficiently using wavelet filters [6]. The scaling basis is constructed from a single function, which is called the scaling function or father function. The scaling basis consists of discrete translates of a dyadic (power of 2) scale transformed copy of the original scaling function. The scale, which is called the fine scale, is determined by the application. The wavelet basis is also constructed from a single function, called the mother wavelet. The wavelet basis consists of discrete translations of dyadic scale-transformed copies of the mother wavelet function. These basis functions are called wavelets. This basis includes wavelets on all dyadic scales between the fine scale and a coarse scale, which is also dictated by the application. The wavelet basis is completed by translates of the scaling function on the coarse scale.

The expansion coefficients for a function in the scaling basis are equivalent to weighted averages of the function over the support of the scaling basis functions. The wavelet basis provides an efficient representation to store this information on multiple scales. The principal advantages of the wavelet method are best explained in terms of properties of these two complementary sets of basis functions.

1. The wavelets and scaling basis functions are orthonormal functions with compact support. This reduces global errors when these bases are used to represent local structures.

2. The wavelet and scaling basis functions are related to a single "mother (resp. father) function" by unit translations and dyadic scale changes. This simplifies the computation of the basis functions.

3. Finite linear combinations of the scaling basis functions can locally rep- 
resent low-degree polynomials. This means that kernels which can be locally approximated by low-degree polynomials on the fine scale can be accurately represented by expansions in a scaling basis. This is the defining property of the Daubechies wavelets.

4. Wavelet basis functions are orthogonal to low-degree polynomials. This is the reason why the kernel in a wavelet basis is sparse. If the kernel can be locally approximated by a polynomial on a sufficiently small region, the matrix elements corresponding to the wavelets with support on that region will be small or zero. In this representation the kernel is accurately represented by a small number of wavelet basis functions on a large scale. Since the wavelet basis is equivalent to the scaling basis, it follows that the wavelet basis can accurately represent kernels that can be locally approximated by low-degree polynomials on the fine scale by sparse matrices.

5. All moments of wavelets or scaling basis functions can be computed exactly and efficiently. These moments are essential for performing the integrals needed for a typical calculation.

6. There is a one-point quadrature rule which integrates the product of second degree local polynomials and scaling basis functions exactly. In our applications, the one-point quadrature rule reduces the Galerkin method, which constructs an approximate matrix equation by projecting the original equation on a subspace spanned by a set of basis functions, to the collocation method, which constructs an approximate matrix equation by demanding that the equation hold on a set of collocation points. The resulting systems of equations share the advantages of both methods.

7. The wavelet transform provides a means to automatically determine the important basis functions. Specifically, it is enough to set all matrix elements of the transformed kernel smaller than a specified minimal size to zero, which yields a sparse matrix.

8. Integrals over the scattering cut can be computed accurately and efficiently using the scaling equation. These integrals can be expressed in terms of non-singular integrals that can be evaluated in terms of the known moments.

9. The integral equation can be solved in the wavelet basis without computing a wavelet or a scaling basis function. This technique exploits many of the properties of the Daubechies wavelets and it saves the computation time involved in evaluating the basis functions.

These features will be explained in more detail in the body of the paper.

Given the many useful properties of Daubechies wavelets, it is natural to ask why they have not been extensively used to treat scattering problems. We speculate that part of the problem is the structure of the wavelets. Wavelets 
are different than the smooth functions that are normally used in numerical analysis. They are finite linear combinations of solutions of a scaling equation, which relates a function on one scale to a linear combination of translates of the same function on a smaller scale. The solution of this scaling equation has a self-similar or fractal structure, which repeats itself on arbitrarily small scales. Functions with fractal structure are not amenable to numerical methods that exploit the smoothness of functions on a sufficiently small scale. Our experience is that typical adaptive quadrature methods are very inefficient when applied to

wavelets. We found this to be especially true for integration over the scattering singularity.

While the fractal structure of wavelets limits the utility of conventional numerical methods, the scaling equation provides an alternative tool, which when properly exploited, can be used to overcome all of the difficulties that arise with conventional methods. An important message in this paper is that success is obtained by embracing the scaling equation.

In preparing this manuscript we found a number of references on wavelets to be useful in understanding how to apply wavelet bases to solve integral equa-

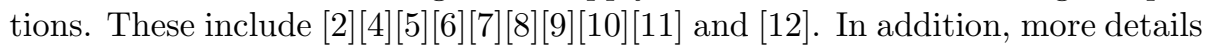
can be found in our web notes [13], which provide a detailed summary of many of the things that we learned from these references. A key element that we believe is new in this paper is the treatment of the singular integral, which has proven to be both stable and accurate. This is also discussed in detail in these notes.

\section{Wavelets}

In this section we define Daubechies' initial family of compactly supported orthogonal functions [5]. The defining property of Daubechies' wavelets is that they are orthogonal to low-degree polynomials.

As mentioned earlier, the basis functions consist of discrete translations and scale transforms of two functions, called the scaling function, which we denote by $\phi(x)$, and mother wavelet function, which we denote by $\psi(x)$. An approximation space has two bases related by a fast orthogonal transformation, called the wavelet transform. One basis consists of translates of the scaling function on a fine scale. The second basis consists of translates of the scaling function on a coarse scale and the wavelets on all dyadic scales between the fine and coarse scale. The first representation will lead to a dense matrix representation of the integral equations while the second results in a sparse matrix representation. The choice of fine scale defines the approximation space; it is determined by the finest structure in the driving term and the kernel of the integral equation.

We begin by discussing the scaling function, $\phi(x)$, which has compact support and integrates to a value of one. To construct the scaling function we utilize two operations, dilation and translation. They are implemented by the 
unitary operators:

$$
\hat{D} \phi(x)=\frac{1}{\sqrt{2}} \phi(x / 2)
$$

which is a dyadic scale transformation and

$$
\hat{T} \phi(x)=\phi(x-1) .
$$

which is a unit translation. The scaling function $\phi(x)$ is the solution of the scaling equation

$$
\hat{D} \phi(x)=\sum_{l=0}^{2 K-1} h_{l} \hat{T}^{l} \phi(x)
$$

with normalization

$$
\int \phi(x) d x=1
$$

Solutions, $\phi(x)$, of the scaling equation with finite $K$ are of special interest because these solutions have compact support in the interval $[0,2 K-1]$ [13].

The scaling equation is a linear renormalization group equation that relates the scaling function on a coarse scale to linear combinations of the scaling function on a fine scale. The coefficients $h_{l}$, called the scaling coefficients, are numerical coefficients which define the type of scaling function. For most computations, a knowledge of the $h_{l}$ 's is all that is needed. In some wavelet literature the normalization convention for the scaling coefficients, $h_{l}$, differs by a factor of $\sqrt{2}$

The scaling basis functions, $\phi_{j, k}(x)$, are defined by taking $j$ dilations and $k$ translations of the scaling function:

$$
\phi_{j, k}(x):=\hat{D}^{j} \hat{T}^{k} \phi(x)=2^{-j / 2} \phi\left(\frac{x}{2^{j}}-k\right) .
$$

Our convention, which follows refs. 44 4 , is that $-\infty<j<\infty$, with decreasing $j$ corresponding to finer scales and increasing $j$ corresponds to coarser scales; some wavelet references use the opposite convention, with increasing $j$ corresponding to finer scales. The scaling function $\phi(x)$ is defined so that the translates of the $\phi_{j, k}(x)$ on a fixed scale $j$ are orthonormal;

$$
\left(\phi_{j, k}, \phi_{j, l}\right)=\delta_{k l}
$$

Self-consistency of the scaling equation (3) and the orthonormality requirement (6) constrain the coefficients $h_{l}$ so that

$$
\sum_{l=0}^{2 K-1} h_{l}=\sqrt{2}, \quad \sum_{l=0}^{2 K-1} h_{l} h_{l-2 n}=\delta_{n 0} .
$$

For $K=1$ these conditions fix the two coefficient $h_{0}$ and $h_{1}$ uniquely. The $K=1$ solution is called the Haar 4 15 scaling function. For larger values of $K$, additional constraints are needed to determine the coefficients $h_{l}$. These 
constraints will be discussed later in the paper. In all cases the, the number of $h_{l}$ 's must be even and the support of the scaling function can be shown [13] to be in the interval $[0,2 K-1]$. The support of $\phi_{j, l}(x)$ has width $2^{j}(2 K-1)$. That the scaling equation has non-trivial solutions with compact support was not known before Daubechies seminal paper 昍.

The subspace of $L^{2}(\mathbb{R})$ spanned by $\phi_{j, k}(x)$ for fixed $j$ is denoted by $\mathcal{V}_{j}$. This space is the approximation space in our calculations. The parameter $j$ defines the scale of these functions, and the size of the support of $\phi_{j, k}(x)$. The scale gets finer as $j$ decreases, and $j$ is determined by the finest scale of the problem. Two important properties of the different subspaces $\mathcal{V}_{j}$ are that

$$
\mathcal{V}_{j-1} \supset \mathcal{V}_{j}
$$

and that $\mathcal{V}_{j}$ becomes dense in $L^{2}(\mathbb{R})$ as $j \rightarrow-\infty$. Equation (8) is a consequence of the scaling equation.

The mother wavelet $\psi(x)$ is defined to be orthogonal to the scaling function and its translates. The mother wavelet is associated with changes in the represented function. It can be visualized as a small wave that integrates to zero. To define the mother wavelet recall Eq. (8). For each $j$ define $\mathcal{W}_{j}$ to be the orthogonal complement of $\mathcal{V}_{j}$ in $\mathcal{V}_{j-1}$ :

$$
\mathcal{V}_{j-1}=\mathcal{W}_{j} \oplus \mathcal{V}_{j}
$$

Orthonormal basis functions of $\mathcal{W}_{j}$ are linear combinations of the scaling basis functions of $\mathcal{V}_{j-1}$. The different basis functions of $\mathcal{W}_{j}$ are discrete translations of a single function. The mother wavelet $\psi(x)$ is the element of $\mathcal{W}_{0}$ defined by the following linear combination of the scaling basis functions on $\mathcal{V}_{-1}$ :

$$
\psi(x):=\hat{D}^{-1} \sum_{l=0}^{2 K-1} g_{k} \hat{T}^{l} \phi(x)
$$

where the coefficients

$$
g_{l}:=(-1)^{l} h_{2 K-1-l}
$$

are fixed by the orthonormality conditions:

$$
\left(\hat{T}^{l} \psi, \hat{T}^{m} \psi\right)=\delta_{l m}, \quad\left(\hat{T}^{l} \psi, \hat{T}^{m} \phi\right)=0 .
$$

The wavelets $\psi_{0, l}(x):=\hat{T}^{l} \psi(x)$ span $\mathcal{W}_{0}$, while

$$
\psi_{j, l}(x):=\hat{D}^{j} \hat{T}^{l} \psi(x) .
$$

is an orthonormal basis for $\mathcal{W}_{j}$. Note that the number of basis functions doubles as $j$ is decreased by one. The support of $\psi_{j, l}(x)$ is identical to the support of $\phi_{j, l}(x)$, which is

$$
\operatorname{supp}\left(\phi_{j, l}(x)\right)=\operatorname{supp}\left(\psi_{j, l}(x)\right)=\left[2^{j} l, 2^{j}(l+2 K-1)\right] .
$$


For a large negative value of $j$ we can iterate (9) to decompose $\mathcal{V}_{j}$ on a fine scale into a direct sum of $\mathcal{W}_{k}$ 's on coarser scales:

$$
\mathcal{V}_{j}=\mathcal{W}_{j+1} \oplus \mathcal{W}_{j+2} \oplus \cdots \oplus \mathcal{W}_{j+m} \oplus \mathcal{V}_{j+m}
$$

In wavelet terminology this is called the multi-resolution decomposition of $\mathcal{V}_{j}$. The right- and left-hand sides of Eq. (14) define two bases for the space $\mathcal{V}_{j}$. The basis on the left consists of just the scaling basis functions $\phi_{j, l}(x)$ on the fine scale " $j$ ". The basis on the right consists of wavelets, $\psi_{k, l}(x)$, on all scales $k$ from the finest $(j+1)$ to the coarsest $(j+m)$ and the scaling basis functions $\phi_{j+m, l}(x)$ on the coarsest scale, $(j+m)$. Note that unlike the spaces $\mathcal{V}_{j}$, the spaces $\mathcal{W}_{j}$ on different scales are orthogonal. This leads to the following alternative representations of functions in $\mathcal{V}_{j}$ :

$$
f(x)=\sum_{l} c_{l} \phi_{j, l}(x)=\sum_{l} d_{l} \phi_{j+m, l}(x)+\sum_{k=j+1}^{j+m} \sum_{l} d_{k, l} \psi_{k, l}(x)
$$

where the expansion coefficients are

$$
c_{l}:=\left(\phi_{j, l}, f\right), \quad d_{l}:=\left(\phi_{j+m, l}, f\right) \quad d_{k, l}:=\left(\psi_{k, l}, f\right) .
$$

It follows from Eqs. (14) and (15) that there is an orthogonal transformation relating the coefficients $c_{l}$ to $d_{l}$ and $d_{k, l}$.

When we approximate a function $f(x)$ as a linear combination the scaling basis functions, $\phi_{j, l}(x)$, and the original function $f(x)$ is approximately constant, $f(x)=f_{l}$, on the support of $\phi_{j, l}(x)$, the expansion coefficients, $c_{l}=\left(f, \phi_{j, l}\right) \approx$ $2^{j / 2} f_{l}$, are up to a scale factor, the value of the function at any point in the support of $\phi_{j, l}(x)$. For a continuous function $f(x)$, and a sufficiently fine scale $j$, it follows that, up to a scale factor, the coefficients $c_{l}$ track the value of $f$ in the sense:

$$
c_{l} \approx 2^{j / 2} f\left(2^{j} l\right) .
$$

The coefficients $c_{l}$ form a very inefficient approximate representation of the function $f(x)$; while the coefficients $d_{l}$ and $d_{k, l}$ form an efficient representation, since many of the $d_{k, l}$ will be small and can be neglected without introducing substantial errors.

The Daubechies wavelets of order $\mathrm{K}$ are defined by the conditions that the mother wavelet satisfy

$$
\int x^{k} \psi(x) d x=0, \quad 0 \leq k \leq K-1 .
$$

Using Eq. (18) gives the additional constraints needed to determine the scaling coefficients $h_{l}$ :

$$
0=\sum_{l=0}^{2 K-1} g_{l} l^{k}=\sum_{l=0}^{2 K-1}(-1)^{l} h_{2 K-1-l} l^{k}, \quad 0 \leq k \leq K-1 .
$$


Table 1: Scaling Coefficients

\begin{tabular}{|l|l|l|l|}
\hline$h_{l}$ & $\mathrm{~K}=1$ & $\mathrm{~K}=2$ & $\mathrm{~K}=3$ \\
\hline$h_{0}$ & $1 / \sqrt{2}$ & $(1+\sqrt{3}) / 4 \sqrt{2}$ & $(1+\sqrt{10}+\sqrt{5+2 \sqrt{10}}) / 16 \sqrt{2}$ \\
$h_{1}$ & $1 / \sqrt{2}$ & $(3+\sqrt{3}) / 4 \sqrt{2}$ & $(5+\sqrt{10}+3 \sqrt{5+2 \sqrt{10}}) / 16 \sqrt{2}$ \\
$h_{2}$ & 0 & $(3-\sqrt{3}) / 4 \sqrt{2}$ & $(10-2 \sqrt{10}+2 \sqrt{5+2 \sqrt{10}}) / 16 \sqrt{2}$ \\
$h_{3}$ & 0 & $(1-\sqrt{3}) / 4 \sqrt{2}$ & $(10-2 \sqrt{10}-2 \sqrt{5+2 \sqrt{10}}) / 16 \sqrt{2}$ \\
$h_{4}$ & 0 & 0 & $(5+\sqrt{10}-3 \sqrt{5+2 \sqrt{10}}) / 16 \sqrt{2}$ \\
$h_{5}$ & 0 & 0 & $(1+\sqrt{10}-\sqrt{5+2 \sqrt{10}}) / 16 \sqrt{2}$ \\
\hline
\end{tabular}

These equations, along with Eq. (7), determine the Daubechies scaling coefficients, $h_{l}$, of order $K$ up to a reversal in the order of the coefficients. Note that the second equation in (7) is actually several equations. There is a different equation for each value of $n$. For the case $K=1,2,3$ these equations can be solved analytically. The solutions are given in Table 1 [6]. For larger values of $K$ these equations can be solved numerically and tables of the scaling coefficients

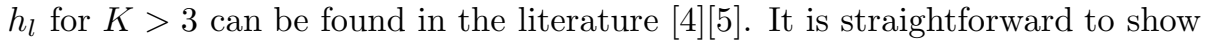
that Eq. 18 holds on all scales:

$$
\int \psi_{j, l}(x) x^{k} d x=0, \quad 0 \leq k \leq K-1 .
$$

Plots of the $K=2$ and $K=3$ scaling function $\phi(x)$ and mother wavelet $\psi(x)$ are shown in Figures 1 and 2. The functions with $K=2$ have support on the interval $[0,3]$ while the $K=3$ functions have support on $[0,5]$. The scaling function and mother wavelet can be distinguished by the conditions that the integral of the scaling function is one while the integral of the mother wavelet is zero. In both figures the compact support and fractal nature of these functions are apparent.

One of the key features of the Daubechies wavelets follows from Eq. (20). Note that in the limit $j \rightarrow-\infty$ the expansion (15) becomes exact. If we expand

$$
x^{k}=\sum_{l} d_{l} \phi_{j, l}(x)+\sum_{k=-\infty}^{j} \sum_{l} d_{k, l} \psi_{k, l}(x),
$$

then the condition (20) gives $d_{k, l}=0$ for $k<K$, or

$$
x^{k}=\sum_{l} d_{l} \phi_{j, l}(x) .
$$

While $x^{k}$ is not a square-integrable function, at any point $x$ the support conditions imply that the above sum has no more than $2 K-1$ non-vanishing terms. It follows that on any compact subset this sum is pointwise identical to $x^{k}$. Note that this is true for any scale $j$. This explains why the scaling function basis and the equivalent wavelet basis can be used to locally represent polynomials. 
Eq. (20) explains why the second basis in Eq. (15) is so efficient. If the function $f(x)$ can be locally approximated by a polynomial of degree $K$ on the support of $\psi_{j, l}(x)$, then the coefficients $d_{k, l}:=\left(\psi_{k, l}, f\right) \approx 0$.

Although the wavelets with higher values of $K$ have some nicer properties, their support and the number of non-zero basis functions at any given point $x$ increases with $K$. Thus, there is a point where the additional smoothness [12] of a higher-order wavelet is balanced against the cost of manipulating functions with larger supports and more scaling coefficients, $h_{l}$. For our application, we believe that the Daubechies $K=3$ wavelet is the optimal choice to balance accuracy with computational speed.

The important observation about the Daubechies wavelets is that once the scaling coefficients $h_{l}$ are determined, all of the calculations can be reduced to manipulations involving just these coefficients. This is done by making judicious use of the scaling equation to derive linear relationships for the quantities that we desire. This method is used to calculate all moments of the scaling and wavelet functions exactly and to construct quadrature rules. It can also be used to compute both $\psi(x)$ and $\phi(x)$ exactly at all dyadic points. Furthermore, the scaling equation leads to the transformation that we need to transform between the scaling basis function representation and the wavelet basis representation. The scaling equation is also used to accurately evaluate the integral of the product of the scaling basis functions and the scattering singularity. Specific applications of the scaling equation that are used to solve for the $K$-matrix are outlined below.

A. Computation of $\phi(x)$ and $\psi(x)$ :

To calculate $\phi(x)$ we use the scaling equation in the form

$$
\phi(x)=\sum_{l=0}^{2 K-1} \sqrt{2} h_{l} \phi(2 x-l) .
$$

Setting $x$ to integer values $n$ gives linear relations among the non-zero $\phi(n)$ 's at integer points:

$$
\phi(n)=\sum_{l=0}^{2 K-1} \sqrt{2} h_{l} \phi(2 n-l) .
$$

These homogeneous equations can be supplemented with the inhomogeneous equation

$$
1=\sum_{n} \phi(n)
$$

to get a linear system of equations for $\phi(x)$ at all integer points. Eq. (25) follows

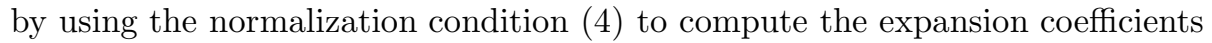
(15) for $f(x)=1$, and evaluating the result at an integer value of $x$.

Given the values $\phi(n)$, recursive use of the scaling equation (23) or (10) gives exact values of $\phi(x)$ or $\psi(x)$ at all dyadic rationals. Since these functions are continuous [0] for $K>1$, and the dyadic rationals are dense in the real numbers, $\phi(x)$ and $\psi(x)$ can be calculated at all points by continuity. While this method 
is exact at dyadic points, it is computationally intensive; however, the method discussed in this paper does not require the evaluation of the wavelets or the scaling basis functions.

\section{B. Moments:}

Moments of the scaling function are defined by

$$
<x^{k}>_{j l}:=\int \phi_{j, l}(x) x^{k} d x
$$

Using

$$
\left(\hat{D}^{j} \hat{T}^{l} \phi, x^{k}\right)=\left(\phi, \hat{T}^{-l} \hat{D}^{-j} x^{k}\right)
$$

these moments can be expressed in terms of the moments $<x^{k}>_{00}$. Using the unitarity of the dilation operator and the scaling equation in

$$
<x^{k}>_{00}=\left(\phi, x^{k}\right)=\left(\hat{D} \phi, \hat{D} x^{k}\right)
$$

gives

$$
<x^{k}>_{00}=\frac{1}{2^{k}} \sum_{l} \frac{h_{l}}{\sqrt{2}} \sum_{m=0}^{k} \frac{k !}{m !(k-m) !} l^{k-m}<x^{m}>_{00} .
$$

This equation can be used to express the higher moments in terms of the lower moments

$$
\begin{gathered}
<x^{k}>_{00}= \\
\frac{1}{2^{k}-1} \sum_{l} \frac{h_{l}}{\sqrt{2}} \sum_{m=0}^{k-1} \frac{k !}{m !(k-m) !} l^{k-m}<x^{m}>_{00}
\end{gathered}
$$

which can be used with the normalization condition (4) to recursively compute moments of any order. Given the moments of the scaling function, Eq. (10) can be used to compute moments of the wavelets.

\section{Quadrature Rules:}

Given a set of moments it is always possible to use them to construct quadrature rules. In most elementary applications a set of quadrature points $\left\{x_{k}\right\}$ is given. The system of linear equations

$$
\sum_{k}\left(x_{k}\right)^{n} w_{k}=<x^{n}>_{00}
$$

is used to solve for the weights $w_{k}$ in terms of the $\left\langle x^{n}>_{00}\right.$ moments. For applications involving a scaling basis function on a fine scale only a small number of quadrature points are needed.

In this paper most of the integrals are performed using a simple and effective one-point quadrature rule that follows from the identity

$$
<x^{2}>_{00}=<x^{1}>_{00}^{2}=\left(\frac{1}{\sqrt{2}} \sum_{l=1}^{2 K-1} l h_{l}\right)^{2},
$$


which holds for the moments of the Daubechies $K>1$ scaling basis functions. In this case one obtains

$$
\int \phi(x) p(x) d x=p\left(<x^{1}>_{00}\right) \quad \text { for } \quad p(x)=a+b x+c x^{2}
$$

which provides a one-point quadrature rule 11, with point $x=\left\langle x^{1}\right\rangle_{00}$ and weight $w=1$, that integrates the product of the polynomial and the scaling function exactly. Translates and dyadic scale transformations of these moments give one-point quadrature rules for each of the $\phi_{j, l}(x)$. In applications we use points $\left\langle x^{1}\right\rangle_{j l}$ and weights $w_{j l}$ for $\phi_{j, l}(x)$ on a small scale $j$ :

$$
<x^{1}>_{j l}=2^{j}\left(<x^{1}>_{00}+l\right) \quad w_{j l}=2^{j / 2}
$$

This quadrature rule is used on the finest scale with the Daubechies $\mathrm{K}=2,3$ scaling basis functions, which can represent local polynomials of degree one and two exactly. For Daubechies wavelets with larger K-values, which can locally represent polynomials of degree $K-1$, multi-point quadratures rules can be constructed which exactly integrate the product of the scaling function with degree $K-1$ polynomials.

\section{Partial Moments:}

In solving integral equations on a finite or semi-infinite interval, it is necessary to calculate integrals where an end-point of the interval is in the support of a scaling basis function [9] 10]. By using the scaling and dilation operators these integrals are simply related to the following integrals:

$$
I_{m+}^{k}:=\int_{0}^{\infty} \hat{T}^{m} \phi(x) x^{k} d x, \quad I_{m-}^{k}:=\int_{-\infty}^{0} \hat{T}^{m} \phi(x) x^{k} d x
$$

and

$$
I_{m n+}^{0}:=\int_{0}^{\infty} \hat{T}^{m} \phi(x) \hat{T}^{n} \phi(x) d x, \quad I_{m n-}^{0}:=\int_{-\infty}^{0} \hat{T}^{m} \phi(x) \hat{T}^{n} \phi(x) d x .
$$

To calculate these quantities one can use the scaling equation in the form (23). This leads to the linear relations

$$
\begin{gathered}
I_{m+}^{k}=2^{-k-1 / 2} \sum_{l=0}^{2 K-1} h_{l} I_{2 m+l,+}^{k} \\
I_{m n+}=\sum_{r=0}^{2 K-1} \sum_{s=0}^{2 K-1} h_{r} h_{s} I_{2 m+r, 2 n+s,+}
\end{gathered}
$$

Because $I_{m+}^{k}=<x^{k}>_{0 m}$ for $m \geq 0$, Eq. (38) becomes a linear system for the unknown partial moments in terms of the full moments. These equations can be solved for the non-trivial $I_{m+}^{k}$. The $I_{m-}^{k}$ are obtained using $I_{m-}^{k}=I_{m}^{k}-I_{m+}^{k}$. Useful examples can be found in [9] and [13. 
For $I_{m n+}$ we have for $m \geq 0$ or $n \geq 0$ :

$$
I_{m n+}=\delta_{m n} .
$$

from the orthonormality of the $\phi_{0 l}(x)$. This leads to a small linear system that relates the unknown integrals $I_{m n+}$ to the known $I_{m n}=\delta_{m n}$. The $I_{m n-}$ can again be obtained by subtraction.

These linear systems can be solved for the non-trivial $I_{m n+}$. The corresponding partial moments on other scales can be obtained from the $I_{m n+}$ using the unitarity of the operator $\hat{D}$ on the half intervals. Partial moments with different endpoints can be obtained by translation and subtraction. These integrals are used to treat endpoint quadratures after the $K$-matrix equation is transformed to a finite interval.

\section{E. Singularity:}

The scaling equation can also be used to solve for the singular integrals of the form

$$
I_{k}^{ \pm}:=\int \frac{\phi(x-k)}{x \pm i 0^{+}} d x .
$$

Application of the scaling equation (3) leads to the linear relations:

$$
I_{k}^{ \pm}:=\sqrt{2} \sum_{l=0}^{2 K-1} h_{l} I_{2 k-l}^{ \pm} .
$$

Using this equation for different values of $k$ leads to a set of linear equations that relate the $I_{k}^{ \pm}$for values of $k$ where the scaling basis function has support containing the singularity to the $I_{k}^{ \pm}$for values of $k$ where the scaling basis function has support far away from the singularity. One more equation relating theses quantities is needed to pick up the contribution from singularity. Specifically we use

$$
1=\sum_{n} \phi_{n}(x)
$$

which follows from Eq. (22) to get:

$$
\mp i \pi=\int_{-a}^{a} \frac{d x}{x \pm i 0^{+}}=\sum_{n} \int_{-a}^{a} \phi_{n}(x) \frac{d x}{x \pm i 0^{+}}=\sum_{n} I_{n: a}^{ \pm},
$$

where

$$
I_{n: a}^{ \pm}=\int_{-a}^{a} \frac{\phi(x-n)}{x \pm i 0^{+}} d x=I_{n}^{ \pm}
$$

except when the points $\pm a$ are in the support of $\phi_{n}(x)$. The values $a$ is chosen to be far from the singularity. For the principal value integral, which is used in this paper, we replace $\mp i \pi$ on the left of Eq. (43) by zero.

The input needed to solve this system are the values of $I_{n: a}^{ \pm}$and $I_{n}^{ \pm}$far from the singularity. For $|n|$ sufficiently large we can calculate these quantities by expanding them in terms of the moments

$$
I_{n}^{ \pm}=\int \frac{\phi(x-n)}{x \pm i 0^{+}} d x=\frac{1}{n} \int \frac{\phi(x)}{1+x / n} d x=\frac{1}{n} \sum_{k=0}^{\infty}\left(\frac{-1}{n}\right)^{k}<x^{k}>_{00}
$$


Table 3 - Integrals over singularity: $\mathrm{K}=2 I_{k}^{ \pm}$

\begin{tabular}{|l|l|l|}
\hline$I_{-1}^{ \pm}$ & -2.779949550280 & $\mp i 4.291495373146$ \\
$I_{-2}^{ \pm}$ & -0.269952669589 & $\pm i 1.149902719556$ \\
\hline
\end{tabular}

Table 4 - Integrals over singularity: $\mathrm{K}=3 I_{k}^{ \pm}$

\begin{tabular}{|l|l|l|}
\hline$I_{-1}^{ \pm}$ & -0.1717835441734 & $\mp i 4.041140804162$ \\
$I_{-2}^{ \pm}$ & -1.7516314066967 & $\pm i 1.212142562305$ \\
$I_{-3}^{ \pm}$ & -0.3025942645356 & $\mp i 0.299291822651$ \\
$I_{-4}^{ \pm}$ & -0.3076858066180 & $\mp i 0.013302589081$ \\
\hline
\end{tabular}

and the partial moments

$$
I_{n: a}^{ \pm}=\int_{-a}^{a} \frac{\phi(x-n)}{x \pm i 0^{+}} d x=\frac{1}{n} \int_{-a}^{a} \frac{\phi(x)}{1+x / n} d x=\frac{1}{n} \sum_{k=0}^{\infty}\left(\frac{-1}{n}\right)^{k}<x^{k}>_{00: a} .
$$

These integrals can be accurately approximated for sufficiently large $|n|$ by truncating the series, (45) and (46), for sufficiently large $|n|$.

Examples of the integrals that overlap the singularity are given in Tables 3 and 4 for the $K=2$ and $K=3$ Daubechies wavelets. The endpoint integrals, where the singularity is located at the boundary of the support, are not singular because of the continuity of $\phi(x)$ for $K>1$. These integrals, and integrals over scaling basis functions close to the singularity are computed using the linear equations (41) and (43). Singular integrals corresponding to different scales are related by the identity

$$
\int \frac{\phi_{j, k}(x) d x}{x \pm i 0^{+}}=2^{j / 2} I_{k}^{ \pm} .
$$

The principal-value integrals are given by the real parts of $I_{k}^{ \pm}$.

\section{Test Problem}

The power of the wavelet method is demonstrated by calculating the $K$-matrix for an s-wave Malfliet-Tjon $\mathrm{V}$ potential. The momentum-space integral equation for the two-body $K$-matrix is

$$
K_{0}\left(p_{1}, p_{2}, p_{0}\right)=v_{0}\left(p_{1}, p_{2}\right)-m \text { P.V. } \int_{0}^{\infty} \frac{v_{0}\left(p_{1}, p^{\prime}\right) p^{\prime 2}}{p^{\prime 2}-p_{0}^{2}} K_{0}\left(p^{\prime}, p_{2}, p_{0}\right) d p^{\prime}
$$

where $m$ is the nucleon mass and P.V. indicates the principal value. The interaction potential is a sum of two Yukawa interactions

$$
v_{0}\left(p_{1}, p_{2}\right):=\sum_{i=1}^{2} \frac{\lambda_{i}}{2 \pi p_{1} p_{2}} \ln \left(\frac{\mu_{i}^{2}+p_{1}^{2}+p_{2}^{2}+2 p_{1} p_{2}}{\mu_{i}^{2}+p_{1}^{2}+p_{2}^{2}-2 p_{1} p_{2}}\right)
$$

with strength and range parameters [14] given in Table 2. 


\section{Table 2: Potential Parameters}

\begin{tabular}{|c|c|c|c|c|}
\hline $1 / m$ & $\lambda_{1}$ & $\mu_{1}$ & $\lambda_{2}$ & $\mu_{2}$ \\
\hline $41.47 \mathrm{MeV} \mathrm{fm}$ & $-570.316 \mathrm{MeV} \mathrm{fm}$ & $1.55 \mathrm{fm}^{-1}$ & $1438.4812 \mathrm{MeV} \mathrm{fm}$ & $3.11 \mathrm{fm}^{-1}$ \\
\hline
\end{tabular}

Test calculations are done for the half on-shell $K$-matrix, $K_{0}\left(p_{1}, p_{2}, p_{0}\right)$, with $p_{2}=p_{0}$, and $p_{0}^{2} / m=10$ and $80 \mathrm{MeV}$.

\section{Wavelet Techniques for Singular Integral Equa- tions}

We solve the integral equation (48) by transforming the half-interval $[0, \infty)$ to a finite interval $[-a, b]$ with the singularity $p_{0}$ transformed to the origin.

For fixed values of $p_{2}$ and $p_{0}$ define

$$
\begin{gathered}
f\left(p_{1}\right):=K\left(p_{1}, p_{2}, p_{0}\right) \\
g\left(p_{1}\right):=v_{0}\left(p_{1}, p_{2}\right) .
\end{gathered}
$$

The fixed variables $p_{2}$ and $p_{0}$, which are just parameters in the integral equation, are suppressed in this notation.

If we also define the non-singular part of the kernel

$$
L\left(p_{1}, p_{2}\right):=m \frac{v_{0}\left(p_{1}, p_{2}\right) p_{2}^{2}}{p_{2}+p_{0}},
$$

the integral equation for the $K$-matrix has the form

$$
f\left(p_{1}\right)=g\left(p_{1}\right)-\mathrm{P} . \mathrm{V} \cdot \int_{0}^{\infty} \frac{L\left(p_{1}, p^{\prime}\right)}{p^{\prime}-p_{0}} f\left(p^{\prime}\right) d p^{\prime} .
$$

To transform $[0, \infty)$ to the interval $[-a, b]$ we use the following map, which also maps the singularity at $p_{0}$ to zero:

$$
\begin{gathered}
p=p(u):=p_{0} \frac{b}{a} \frac{a+u}{b-u} \quad u=u(p):=\frac{a b\left(p-p_{0}\right)}{a p+p_{0} b} \\
d p=p_{0} \frac{b}{a} \frac{(b+a)}{(b-u)^{2}} d u . \\
\frac{1}{p-p_{0}}=\frac{a(b-u)}{(a+b) p_{0}} \frac{1}{u} .
\end{gathered}
$$

Let

$$
\tilde{g}(u):=g\left(p_{0} \frac{b}{a} \frac{a+u}{b-u}\right),
$$




$$
\tilde{f}(u):=f\left(p_{0} \frac{b}{a} \frac{a+u}{b-u}\right)
$$

and

$$
\tilde{L}(u, v)=L\left(p_{0} \frac{b}{a} \frac{a+u}{b-u}, p_{0} \frac{b}{a} \frac{a+v}{b-v}, p_{0}\right) \frac{b}{(b-v)},
$$

where the factor $b /(b-v)$ is the product of the Jacobian and the residue of the transformed singularity

$$
\frac{b}{b-v}=p_{0} \frac{b}{a} \frac{(b+a)}{(b-v)^{2}} \frac{a(b-v)}{(a+b) p_{0}} .
$$

The integral equation in the $u, v$ variables has the form

$$
\tilde{f}(u)=\tilde{g}(u)+\int_{-a}^{b} \frac{\tilde{L}(u, v)}{v} \tilde{f}(v) d v
$$

To solve this we represent $\tilde{f}(u)$ using the scaling basis on a sufficiently fine scale $j$ :

$$
\tilde{f}(u) \approx \sum_{n} \tilde{f}_{n} \phi_{j, n}(u) .
$$

Inserting the expansion (62) in the integral equation (61) gives

$$
\sum_{n} \phi_{j, n}(u) \tilde{f}_{n}=\tilde{g}(u)+\sum_{n} \int_{-a}^{b} \frac{\tilde{L}(u, v)}{v} \phi_{j, n}(u) d v \tilde{f}_{n} .
$$

To get a linear system for the coefficients $f_{n}$ we multiply the above equation by $\phi_{j, m}(u)$ and integrate from $-a$ to $b$ :

$$
\sum_{n} N_{m n} \tilde{f}_{n}=\tilde{g}_{m}+\sum_{n} \int_{-a}^{b} \phi_{j, m}(v) \frac{\tilde{L}(u, v)}{v} \phi_{j, n}(u) d u d v \tilde{f}_{n},
$$

where

$$
N_{m n}:=\int_{-a}^{b} \phi_{j, m}(u) \phi_{j, n}(u) d u
$$

The matrix $N_{m n}$ is $\delta_{m n}$ except when the support of $\phi_{j, m}(u)$ and $\phi_{j, n}(u)$ contains $-a$ or $b$. This is expressed in the form

$$
N_{m n}=\delta_{m n}+\Delta_{m n} .
$$

The integrals $N_{m n}$ can be computed exactly using Eq. (36-39). The integrals, $N_{m n}$, the singular integrals, $I_{m}^{ \pm}$, as well as the moments, partial moments, and quadrature points can be calculated once and stored for later use.

The vector $\tilde{g}_{m}$ is given by

$$
\tilde{g}_{m}=\int_{-a}^{b} \tilde{g}(u) \phi_{j, m}(u) d u
$$


which is computed using the one-point quadrature when $-a$ or $b$ is not in the support of $\phi_{j, m}(u)$. In the case that $-a$ or $b$ is in the support of $\phi_{j, m}$ we use the partial moments (35) to construct endpoint quadrature rules (31). We use $K+1$ transformed Gauss-Legendre points. The weights are determined by solving a linear equation determined by the requirement that the quadrature rule reproduces the lowest-order partial moments up to order $K$.

The matrix elements of the kernel are evaluated using

$$
\begin{aligned}
\tilde{L}_{m, n}:=\int_{-a}^{b} \phi_{j, m}(v) \frac{\tilde{L}(v, u)}{u} \phi_{j, n}(u) d u d v \\
=\int_{-a}^{b} \phi_{j, m}(v) \frac{\tilde{L}(v, u)-\tilde{L}(v, 0)}{u} \phi_{j, n}(u) d u d v \\
\quad+\int_{-a}^{b} \phi_{j, m}(v) \tilde{L}(v, 0) d v \int_{-a}^{b} \frac{\phi_{j, n}(u)}{u} d u .
\end{aligned}
$$

For most basis functions the $v$ integral is done using the one-point quadrature rule. This is also the case in Eq. (67) for the " $u$ " integral and for the integral over the subtracted term in Eq. (68). The quadratures used for the endpoints of $\hat{g}_{m}$ are used to integrate scaling functions with support on the endpoints of the integral. The singular " $u$ " integral on the last line of Eq. (68) is done using the method discussed in the previous section.

The resulting equation has the form

$$
\tilde{f}_{m}=\tilde{g}_{m}+\sum_{n}\left(\tilde{L}_{m, n}-\Delta_{m, n}\right) \tilde{f}_{n}
$$

The coefficients $\tilde{f}_{m}$ obtained by solving this matrix equation can be used to calculate a refined solution, which is constructed by substituting the series solution into the right-hand side of the integral equation. If the integrals are evaluated following the same procedure used in the integral equations, the solution $\tilde{f}(u)$ can be obtained for any value of $u$ without having to calculate the basis functions $\phi_{j, m}(u)$. This is significant because the evaluation of the basis functions is computationally intensive. The refined solution, $f(u)$, can be used to evaluate the half on-shell $K$-matrix at any point $p$ by using the inverse of (58):

$$
K\left(p, p_{0}, p_{0}\right)=f(p)=\tilde{f}\left(\frac{a b\left(p-p_{0}\right)}{a p+p_{0} b}\right)
$$

The calculation outlined above, where the transformed K-matrix equation is solved in the scaling function basis, has a full kernel matrix. While the treatment of the singular integral is very stable and accurate, the method does not lead to a reduction in the size of the matrix equations when compared to calculations using splines or other numerical techniques. In the next section we show that if Eq. (69) is transformed to an equivalent equation in the wavelet basis, that the resulting kernel matrix is well approximated by a sparse matrix, which leads to a considerable reduction in the scale of the numerical calculation. We also show that the wavelet transform can be implemented by an efficient algorithm that uses a simple filter on multiple scales. 


\section{Wavelet Transform}

The computation done in the previous section leads to a linear system with a large dense matrix. The solution is a set of numerical coefficients $\tilde{f}_{l}=c_{j, l}$ of the approximate solution in the scaling function basis for a fine scale $j$. As mentioned earlier, there are two equivalent representations for the solution $\tilde{f}(u)$ given in Eq. (15). The wavelet transform for a problem with $2^{N}$ basis functions maps coefficients of the basis functions on model space $\mathcal{V}_{j}$ to coefficients of the basis functions on the equivalent space $\mathcal{W}_{j+1} \oplus \cdots \mathcal{W}_{j+N} \oplus \mathcal{V}_{j+N}$.

The form of the transformation is determined by applying the Eqs. (3) and (10) to map

$$
\mathcal{V}_{l} \rightarrow \mathcal{V}_{l+1}
$$

and

$$
\mathcal{V}_{l} \rightarrow \mathcal{W}_{l+1}
$$

This is done $N$ times, successively applying Eqs. (3) and (10) to $\mathcal{V}_{j}, \mathcal{V}_{j+1} \cdots \mathcal{V}_{j+N-1}$.

The coefficients on the next (coarser) scale are obtained using

$$
\begin{aligned}
& c_{j+1, m}=\sqrt{2} \sum_{l} h_{l} c_{j, m+l} \\
& d_{j+1, m}=\sqrt{2} \sum_{l} g_{l} c_{j, m+l} .
\end{aligned}
$$

Because only a finite number of coefficients are considered, there is some ambiguity in how to treat the coefficients that appear on the right-hand side of these equations, which are out of range. The simplest method to treat these terms is to use a periodic wrap-around condition:

$$
c_{j+k, l+2^{N-k}} \rightarrow c_{j+k, l},
$$

which is applied at each level, $k$. Since the result is an orthogonal transformation of the original basis, this choice does not affect the solution. It will have a small edge effect on the structure of the resulting sparse matrix.

The structure of the successive transformations is illustrated for the case $j=-3$ and $N=3$ by the following diagram. In this diagram $c_{j, m}$ represents the coefficients of the basis functions in $\mathcal{V}_{j}$ and $d_{j, m}$ represents the coefficients of the basis functions in $\mathcal{W}_{j}$. The successive transformations act on $c_{-3, l}$ as follows:

$$
\left(\begin{array}{l}
c_{-3,1} \\
c_{-3,2} \\
c_{-3,3} \\
c_{-3,4} \\
c_{-3,5} \\
c_{-3,6} \\
c_{-3,7} \\
c_{-3,8}
\end{array}\right) \rightarrow\left(\begin{array}{l}
c_{-2,1} \\
c_{-2,2} \\
c_{-2,3} \\
c_{-2,4} \\
d_{-2,1} \\
d_{-2,2} \\
d_{-2,3} \\
d_{-2,4}
\end{array}\right) \rightarrow\left(\begin{array}{l}
c_{-1,1} \\
c_{-1,2} \\
d_{-1,1} \\
d_{-1,2} \\
d_{-2,1} \\
d_{-2,2} \\
d_{-2,3} \\
d_{-2,4}
\end{array}\right) \rightarrow\left(\begin{array}{l}
c_{-0,1} \\
d_{-0,1} \\
d_{-1,1} \\
d_{-1,2} \\
d_{-2,1} \\
d_{-2,2} \\
d_{-2,3} \\
d_{-2,4}
\end{array}\right)
$$


This transform can be implemented efficiently. Since it is an orthogonal transformation, if it is expressed in matrix form, the inverse is just the transpose. Both the wavelet transform and its inverse can be implemented in $O(N)$ [7] steps.

The advantage of using the wavelet basis is that the coefficients $d_{j, k}$ in Eq. (15) will be small if $\int \tilde{f}(u) \psi_{j, k}(u) d u \approx 0$. This will be true, by virtue of Eq. (20), whenever $\tilde{f}(u)$ can be locally approximated by a polynomial of degree less than $K$ on the support of $\psi_{j, k}(u)$. A practical rule of thumb states that the number of non-zero matrix elements grows like $10 N \log (1 / \epsilon)$ [6], where $N$ is the size of one dimension of the matrix, and $\epsilon$ is the absolute value of the ratio of size of the smallest matrix element retained to the largest matrix element. This behavior is consistent with our observations.

In Figure 3 a simple example is used to illustrate how structure appears on different scales once the wavelet transform is applied. In this case a product of two Gaussian functions with displaced broad and narrow peaks is expressed in a $\mathrm{K}=3$ Daubechies scaling basis. The result is transformed with the wavelet transform and the strength of the coefficients in the wavelet basis are studied. The figure shows the coefficients of the scaling functions on the coarse scale give a low-resolution large-scale representation of the original function. The coefficients of the wavelets on different scales builds the fine structure. Note the that scales on the vertical axes differ in each frame. It is also interesting to see how the wavelet transform automatically finds the parts of the original function with structure.

To fully use the wavelet transform, the number of basis functions should be a power of two. In this paper the choice of $-a$ and $b$ is adjusted so that the number of basis functions is a power of two. By translating the interval $[-a, b]$ it is also possible to control the number of basis functions with support on either side of the singularity at zero.

In applications the wavelet transformation is applied to the driving term and to the rows and columns of the kernel of the integral equation. This leads to an equivalent linear system with respect to the transformed basis. In this new basis we expect the matrix of the kernel to be the sum of a sparse matrix and a matrix with small norm. Ignoring the small normed matrix leads to an approximate linear system with a sparse kernel.

This approximate solution can be transformed back to the scaling representation and substituted back into the integral equation. Evaluating the integrals using the one-point quadrature and methods discussed previously, gives the refined solution, constructed without computing the wavelets or scaling basis functions.

\section{Results}

Initial calculations are performed using $\mathrm{K}=2$ and $\mathrm{K}=3$ Daubechies wavelets using $N$ scaling basis functions, up to $N_{\max }=2^{9}=512$. The finest resolution corresponds to $j:=J=-7$. The values of $a$ and $b$ are given by $a=1$ and 
$b=-a+(N-2 K+2) 2^{J}$. The solutions are represented directly in terms of a series expansion in the scaling function basis and using this expansion in the integral equation to construct the refined solution. The refined solution has the advantages that the fractal structure of the basis does not appear in the solution and the basis functions do not have to be evaluated. These calculations were checked against conventional differential and integral equation programs, and the agreement was excellent.

Calculations were done for the half on-shell K-matrix at 10 and $80 \mathrm{MeV}$. Tables 5-8 show the values of the refined on-shell K-matrix elements at 10 and $80 \mathrm{MeV}$ for the Daubechies $K=2$ and $K=3$ scaling function bases for different resolutions $j$ (approximation spaces $\mathcal{V}_{j}$ ). These tables illustrate the convergence of a full calculation as the resolution is increased. In each successive calculation the resolution is increased by a factor of 2 .

The convergence for the $K=2$ and $K=3$ calculations are compared for different values of $j=J$, which defines the smallest scale, in Tables 9-10. It is apparent that both methods are accurate, but the $K=3$ Daubechies basis gives better results for the same number of basis functions.

In this paper the refined solution with $N=2^{9}$ scaling basis functions serves as the benchmark calculation that is used to test the approximate wavelet calculations with sparse matrices. In general, the method leads to accurate and stable solutions. This solution is converged to the accuracy expressed in the tables and agrees with calculations using other methods.

Applying the wavelet transform to the integral equation and throwing away all matrix elements of the kernel smaller than a given size leads to approximate equations with sparse matrices. Tables 11-13 compare the solution of different sparse matrix approximations to the full 512 basis function calculation for the $K=2$ and $K=3$ Daubechies basis functions at 10 and $80 \mathrm{MeV}$. The first column in each of these tables indicates that matrix elements with an absolute value smaller than $\epsilon$ times the absolute value of the largest matrix elements are set to zero. The second column indicates the percentage of the 512 matrix elements that are non-zero in the sparse matrix approximation. The third column indicates the on-shell value of the $K$-matrix obtained by applying the integral equation refinement to the sparse matrix solution. Column five shows the relative error at the on-shell point and column six shows the mean square error of solution to the integral equation over the whole domain defined by:

$$
\left|\frac{\tilde{f}(0)-\tilde{f}_{\epsilon}(0)}{\tilde{f}(0)}\right|
$$

and

$$
\frac{\left.\int\left(\tilde{f}(u)-\tilde{f}_{\epsilon}(u)\right)^{2} d u\right)^{1 / 2}}{\left(\int \tilde{f}^{2}(u) d u\right)^{1 / 2}} .
$$

The power of the wavelet method is apparent in the $K=3$ Daubechies basis. Tables 12 and 14 show, for the case $\epsilon=10^{-6}$, that about $96 \%$ of the matrix elements are eliminated relative to the full calculations. The on-shell error is a 
few parts in $10^{5}$ for both energies. Even more impressive is the fact that the mean-square error for the half-shell $K$-matrix is also about the same order of magnitude.

Figure 4 shows the transformed half on-shell K-matrix, $\tilde{f}(u)$ at $80 \mathrm{MeV}$ using the sparse matrix approximation with $\epsilon=10^{-6}$. This figure is a plot of the series solution, which is indistinguishable from the refined solution. The smoothness of the series solution is apparent from this figure. While in Figure 5 we show the half on-shell K-matrix, $f\left(p_{1}\right)$, at $80 \mathrm{MeV}$ using the sparse matrix approximation with $\epsilon=10^{-6}$. This plot shows the refined solution, which is obtained by substituting the series solution back in the integral equation. As in the transformed case, this is indistinguishable from the series solution. Figure 6 provides a graphic illustration of how the non-zero terms of the sparse kernel are distributed for the case $\epsilon=10^{-6}$. What is relevant is that the two dimensional plot is dominated by a number of one-dimensional structures. The vertical bands in this matrix correspond to basis functions with support near the singularity, where we expect significant structure. The different diagonal bands arise because local structures in the kernel can couple wavelets on several scales.

While it is possible to calculate perturbative corrections to approximate solutions, it make more sense calculate the matrix equation in the scaling basis at a finer scale, and use the wavelet transform to get a better approximation. This is because the matrix with small norm, that is needed to compute perturbative corrections, is not sparse.

The tables and calculations indicate that wavelet methods provide a potentially powerful technique for reducing the size of momentum-space scattering integral equations. In this paper we did this by transforming a dense kernel using the wavelet transform. These operations can be done very efficiently, resulting in a matrix which is the sum of a sparse matrix and a matrix with small norm.

Solving the linear system using only the sparse matrix leads to accurate solutions. In our application a decrease in the size of the matrix by a factor of 20 led to an error of a few parts in $10^{5}$ for the Daubechies $K=3$ wavelets.

The speed of our computations is helped by using the one-point quadrature, which leads to accurate answers when used with the Daubechies $K=2$ or 3 wavelets. Another nice feature of the wavelet method is that it automatically puts basis functions at places where the kernel has structure, as compared to splines, where the placement of the knots must be done by hand.

\section{Summary}

In this paper we adapted numerical methods based on wavelets to calculate the K-matrix for a Malfliet-Tjon V potential in momentum space. The motivation for this work was to determine if numerical methods based on wavelets could be used to reduce the size of the matrices that occur in numerical implementations of the momentum-space integral scattering equations. 
There are three reasons for investigating this problem:

1. Relativistic formulations of the few-body problem are naturally formulated in momentum space. This is because the momentum operators are infinitesimal generators of the Poincaré group. Operators, like Wigner rotations, have simple forms in momentum space.

2. Boundary conditions and scattering asymptotic conditions can be cleanly formulated in momentum-space compact-kernel integral equations.

3. The kernel matrix that appears in most numerical implementations of momentum-space scattering equations is full. The size of the kernel matrix is also large for few-body calculations with realistic interactions.

We found that the matrix representation of the momentum-space scattering integral equations in the wavelet basis leads to a linear system with a sparse approximate kernel matrix. This leads to a significant reduction in the size of the dynamical calculation.

Our applications used the Daubechies $K=2$ or $K=3$ wavelets. For each value of $K$ there were two bases, the scaling function basis and the wavelet basis, which were related by a fast orthogonal transform, called the wavelet transform. The $K=2$ bases can locally represent lines of arbitrary slope on subintervals corresponding to the finest scale. The $K=3$ bases can locally represent quadratic curves on subintervals corresponding to the finest scale. Our observations indicate that we obtain better accuracy with the same number of basis functions using the $K=3$ basis than with the $K=2$ basis without a significant increase in computational complexity. While it is possible to use a basis with a higher $K$ value, in order to take advantage of the additional capabilities of the basis functions, the one-point quadrature would have to be replaced by a multi-point quadrature. The additional benefit is not worth the complexity and increased computational effort required by the additional quadrature points.

One potential difficulty with using wavelet methods in numerical calculations is the self-similar structure of the basis functions. Functions that do not become smooth on a sufficiently small scale are not amenable to conventional numerical methods. Initially, we found that this property caused numerical challenges. To overcome these problems we utilized the scaling equation to exactly calculate moments of the scaling basis functions or a wavelets times a polynomial. We found that by using these moments and the scaling equation we were able to exactly compute overlap integrals, to accurately evaluate integrals over the scattering singularity, and to exactly compute endpoint overlap integrals. It is only after using the scaling equation to do all of the required quadratures that we were able to obtain stable and accurate answers. While many of the techniques discussed in this paper are standard wavelet procedures, the method for treating the scattering singularity seems to be new to this paper.

As a basis for solving integral equations, the scaling function basis has many of the same advantages as a spline basis. The most important common features are the compact support and the ability to exactly represent polynomials on 
small scales. This means the they can be used to efficiently approximate local structures. The scaling function basis has the additional feature that the functions are orthonormal. In addition, for the $K=2,3$ Daubechies wavelets, the one-point quadrature can be used to exactly compute the integral of the product of a degree two polynomial and the scaling basis function on the support of the scaling basis function. By employing the one-point quadrature rule, the matrix formulation of the integral equation can be computed very quickly. While the wavelets and scaling basis functions are computationally intensive to compute, the one-point quadrature can be used to replace the evaluations of these functions if the series solutions are substituted back in the integral equation. This method, leads to our refined solution. The only input that is needed are the scaling coefficients, $h_{l}$, which are known analytically for the $K=2$ and $K=3$ wavelets.

In working on this problem, we were able to find a number of previous applications of wavelet methods to integral equations; but were unable find applications that treated integral equations with the singular kernels of momentum space scattering equations. We found that the required integrals could be accurately computed using the scaling equation. The method has proved to be very stable. It has the advantage that it reduced the calculation of these integrals to solving a small system of linear equations, the results of which can be stored for later calculations.

The real advantage of the wavelet method over the conventional methods occurs after the wavelet transform is applied to the K-matrix equation in the scaling function basis. The kernel matrix in the wavelet basis can be expressed as the sum of sparse matrix and a matrix of small norm. Ignoring the small matrix leads to an approximate solution. The case featured in Figure 6, used Daubechies $K=3$ wavelets. The sparse matrix approximation used in Figure 6 led to a $96 \%$ reduction in the number of non-zero matrix elements. This was done with a dimensionless mean square error of a few parts in $10^{5}$

The beauty of the wavelet transform is that it is fast, and it automatically finds the basis functions needed to represent the small scale structure of the solution.

In this paper we have exploited the scaling equation to formulate a powerful method for solving momentum space scattering integral equations. While we limited our application to what was necessary to solve the scattering integral equations, it is apparent that there are many thing that can be computed exactly or to a very high accuracy with the scaling equation. For example, it is possible to exactly integrate products of several scaling functions and powers of the variable. This suggests that these methods could be very useful for solving a large class of physics problems.

Our conclusion is that wavelet bases provide a powerful method for solving momentum-space scattering integral equations. The required matrix elements are easy to compute, and the wavelet transform can be implemented very efficiently, leading to an approximate linear system with a sparse matrix. 
Table 5 - Dependence on number of basis functions: $K=2, E=10$ $\mathrm{MeV}$

\begin{tabular}{|l|l|l|l|}
\hline$-\mathrm{J}$ & $\mathrm{N}$ & series on-shell & refined on-shell \\
\hline 3 & 32 & -124.681226 & -124.401416 \\
4 & 64 & -124.924473 & -124.853374 \\
5 & 128 & -124.984907 & -124.967026 \\
6 & 256 & -124.999853 & -124.995372 \\
7 & 512 & -125.003567 & -125.002445 \\
\hline
\end{tabular}

Table 6 - Dependence on number of basis functions: $\mathrm{K}=3, \mathrm{E}=10 \mathrm{MeV}$

\begin{tabular}{|l|l|l|l|}
\hline$-\mathrm{J}$ & $\mathrm{N}$ & series on-shell & refined on-shell \\
\hline 3 & 32 & -125.051451 & -125.034060 \\
4 & 64 & -125.007967 & -125.006049 \\
5 & 128 & -125.005171 & -125.004948 \\
6 & 256 & -125.004847 & -125.004820 \\
7 & 512 & -125.004806 & -125.004803 \\
\hline
\end{tabular}

Table 7 - Dependence on number of basis functions: $K=2, E=80 \mathrm{MeV}$

\begin{tabular}{|l|l|l|l|}
\hline$-\mathrm{J}$ & $\mathrm{N}$ & series on-shell & refined on-shell \\
\hline 3 & 32 & -6.53375948 & -6.38393342 \\
4 & 64 & -6.45483277 & -6.41711946 \\
5 & 128 & -6.43490787 & -6.42555390 \\
6 & 256 & -6.42998750 & -6.42766546 \\
7 & 512 & -6.42877076 & -6.42819277 \\
\hline
\end{tabular}

Table 8 - Dependence on number of basis functions: $\mathrm{K}=3, \mathrm{E}=80 \mathrm{MeV}$

\begin{tabular}{|l|l|l|l|}
\hline$-\mathrm{J}$ & $\mathrm{N}$ & series on-shell & refined on shell \\
\hline 3 & 32 & -6.44161445 & -6.43154124 \\
4 & 64 & -6.42926712 & -6.42868443 \\
5 & 128 & -6.42842366 & -6.42840177 \\
6 & 256 & -6.42837147 & -6.42837210 \\
7 & 512 & -6.42836848 & -6.42836877 \\
\hline
\end{tabular}

Table 9 - Refined on-shell values, $\mathrm{E}=10 \mathrm{MeV}$

\begin{tabular}{|l|l|l|}
\hline $\mathrm{N}$ & $\mathrm{K}=2$ on-shell & $\mathrm{K}=3$ on-shell \\
\hline 32 & -124.401416 & -125.034060 \\
64 & -124.853374 & -125.006049 \\
128 & -124.967026 & -125.004948 \\
256 & -124.995372 & -125.004820 \\
512 & -125.002445 & -125.004803 \\
\hline
\end{tabular}


Table 10 - Refined on-shell values, $\mathrm{E}=80 \mathrm{MeV}$

\begin{tabular}{|l|l|l|}
\hline $\mathrm{N}$ & $\mathrm{K}=2$ on-shell & $\mathrm{K}=3$ on-shell \\
\hline 32 & -6.38393342 & -6.43154124 \\
64 & -6.41711946 & -6.42868443 \\
128 & -6.42555390 & -6.42840177 \\
256 & -6.42766546 & -6.42837210 \\
512 & -6.42819277 & -6.42836877 \\
\hline
\end{tabular}

Table 11 - Sparse Matrix Convergence: $\mathrm{K}=2, \mathrm{E}=10 \mathrm{MeV}, \mathrm{J}=\mathbf{- 7}$

\begin{tabular}{|l|l|l|l|l|}
\hline$\epsilon$ & percent & on-shell value & on-shell error & mean-square error \\
\hline 0 & 100 & -125.00245 & 0 & 0 \\
$10^{-9}$ & 34.24 & -125.00244 & $6.94 \times 10^{-9}$ & $8.76 \times 10^{-9}$ \\
$10^{-8}$ & 20.8 & -125.00244 & $3.82 \times 10^{-8}$ & $7.81 \times 10^{-8}$ \\
$10^{-7}$ & 12.16 & -125.00440 & $1.57 \times 10^{-5}$ & $1.63 \times 10^{-5}$ \\
$10^{-6}$ & 6 & -124.99271 & $7.78 \times 10^{-5}$ & $9.53 \times 10^{-5}$ \\
$10^{-5}$ & 2.61 & -124.96069 & .000334 & .000435 \\
$10^{-4}$ & 1.16 & -124.47119 & .00425 & .00457 \\
$10^{-3}$ & .55 & -120.42759 & .0366 & .0415 \\
$10^{-2}$ & .31 & -116.14345 & .0709 & .154 \\
\hline
\end{tabular}

Table 12 - Sparse Matrix Convergence: $\mathrm{K}=3, \mathrm{E}=10 \mathrm{MeV}, \mathrm{J}=-7$

\begin{tabular}{|l|l|l|l|l|}
\hline$\epsilon$ & percent & on-shell value & on-shell error & mean-square error \\
\hline 0 & 100 & -125.00480 & 0 & 0 \\
$10^{-9}$ & 17.78 & -125.00480 & $1.05 \times 10^{-8}$ & $2.56 \times 10^{-8}$ \\
$10^{-8}$ & 11.38 & -125.00480 & $5.14 \times 10^{-8}$ & $2.44 \times 10^{-7}$ \\
$10^{-7}$ & 6.6 & -125.00475 & $4.49 \times 10^{-7}$ & $1.88 \times 10^{-6}$ \\
$10^{-6}$ & 3.76 & -125.00269 & $1.69 \times 10^{-5}$ & $2.08 \times 10^{-5}$ \\
$10^{-5}$ & 2.14 & -124.99030 & .000116 & .000228 \\
$10^{-4}$ & 1.24 & -124.85112 & .00123 & .00217 \\
$10^{-3}$ & .72 & -123.82508 & .00944 & .0117 \\
$10^{-2}$ & .38 & -125.25766 & .00202 & .128 \\
\hline
\end{tabular}

Table 13 - Sparse Matrix Convergence: $\mathrm{K}=\mathbf{2}, \mathrm{E}=\mathbf{8 0}$, MeV J=-7

\begin{tabular}{|l|l|l|l|l|}
\hline$\epsilon$ & percent & on-shell value & on-shell error & mean-square error \\
\hline 0 & 100 & -6.4281928 & 0 & 0 \\
$10^{-9}$ & 38.25 & -6.4281928 & $4.16 \times 10^{-11}$ & $6.30 \times 10^{-9}$ \\
$10^{-8}$ & 23.59 & -6.4281927 & $1.54 \times 10^{-8}$ & $1.13 \times 10^{-7}$ \\
$10^{-7}$ & 13.84 & -6.4281936 & $1.30 \times 10^{-7}$ & $2.11 \times 10^{-6}$ \\
$10^{-6}$ & 6.89 & -6.4282684 & $1.18 \times 10^{-5}$ & $3.87 \times 10^{-5}$ \\
$10^{-5}$ & 2.91 & -6.4286669 & $7.38 \times 10^{-5}$ & .000211 \\
$10^{-4}$ & 1.18 & -6.4286269 & $6.75 \times 10^{-5}$ & .00166 \\
$10^{-3}$ & .55 & -6.4083948 & .00308 & .0113 \\
$10^{-2}$ & .3 & -6.3206920 & .0167 & .101 \\
\hline
\end{tabular}


Table 14 - Sparse Matrix Convergence: $\mathrm{K}=3, \mathrm{E}=80$, MeV J=-7

\begin{tabular}{|l|l|l|l|l|}
\hline$\epsilon$ & percent & on-shell value & on-shell error & mean-square error \\
\hline 0 & 100 & -6.4283688 & 0 & 0 \\
$10^{-9}$ & 19.99 & -6.4283688 & $1.52 \times 10^{-10}$ & $1.20 \times 10^{-8}$ \\
$10^{-8}$ & 12.94 & -6.4283690 & $3.44 \times 10^{-8}$ & $2.06 \times 10^{-7}$ \\
$10^{-7}$ & 7.42 & -6.4283703 & $2.33 \times 10^{-7}$ & $1.87 \times 10^{-6}$ \\
$10^{-6}$ & 4.08 & -6.4283333 & $5.51 \times 10^{-6}$ & $4.38 \times 10^{-5}$ \\
$10^{-5}$ & 2.22 & -6.4278663 & $7.82 \times 10^{-5}$ & .000994 \\
$10^{-4}$ & 1.21 & -6.4244211 & .000614 & .00845 \\
$10^{-3}$ & .67 & -6.4154328 & .00201 & .0229 \\
$10^{-2}$ & .34 & -6.2935398 & .021 & .102 \\
\hline
\end{tabular}

\section{References}

[1] R. A. Malfliet, J. A. Tjon: Nucl. Phys. A127,161(1969)

[2] Barbara Burke Hubbard: The World According To Wavelets. Wellesly, MA: A. K. Peters. 1996

[3] See http://www.jpeg.org

[4] Ingrid Daubechies: Comm. on Pure and Applied Mathematics Vol. XLI, 909-996(1988)

[5] Ingrid Daubechies: Ten Lecture on Wavelets. Philadelphia, PA: SIAM 1992

[6] W. H. Press, S. A. Teukolsky, W. T. Vettering, B. P. Flannery: Numerical Recipes in C, Cambridge University Press 1992

[7] H.L. Resnikoff and R.O. Wells: Wavelet Analysis: The Scalable Structure of Information. New York: Springer-Verlag 1998

[8] G. Strang: Wavelets and Dilation Equations: A Brief Introduction. SIAM Review, 31:4, 614-627 (1989)

[9] W.-C. Shann: Quadrature rules needed in Galerkin-wavelets methods, Proceedings for the 1993 annual meeting of Chinese Mathematics Association. Chiao-Tung Univ Dec (1993). (http://www.math.ncu.edu.tw/ shann/Math/pre.htm])

[10] W.-C. Shann and J.-C. Yan: Quadratures involving polynomials and Daubechies' wavelets. Technical Report 9301. Department of Mathematics, National Central University 1993. (http://www.math.ncu.edu.tw/ shann/Math/pre.htm] )

[11] W. Sweldens and R. Piessens: Quadrature Formulae and Asymptotic Error Expansions for wavelet approximations of smooth functions. SIAM J. Numer. Anal., 31,1240-1264(1994) 
[12] G. Kaiser: A Friendly Guide to Wavelets. Birkhäuser, 1994

[13] B. M. Kessler, G. L. Payne, W. N. Polyzou: Notes on Wavelets, (in preparation)

[14] G. L. Payne, J. L. Friar, B. F. Gibson, and I. R. Afnan, :Phys. Rev. C22, $823(1980)$

[15] A. Haar: Zur Theorie der orthogonalen Funktionen-Systeme: Math. Ann. $69,331(1910)$ 
Figure 1: Daubechies $K=2$ Scaling function and mother wavelet function

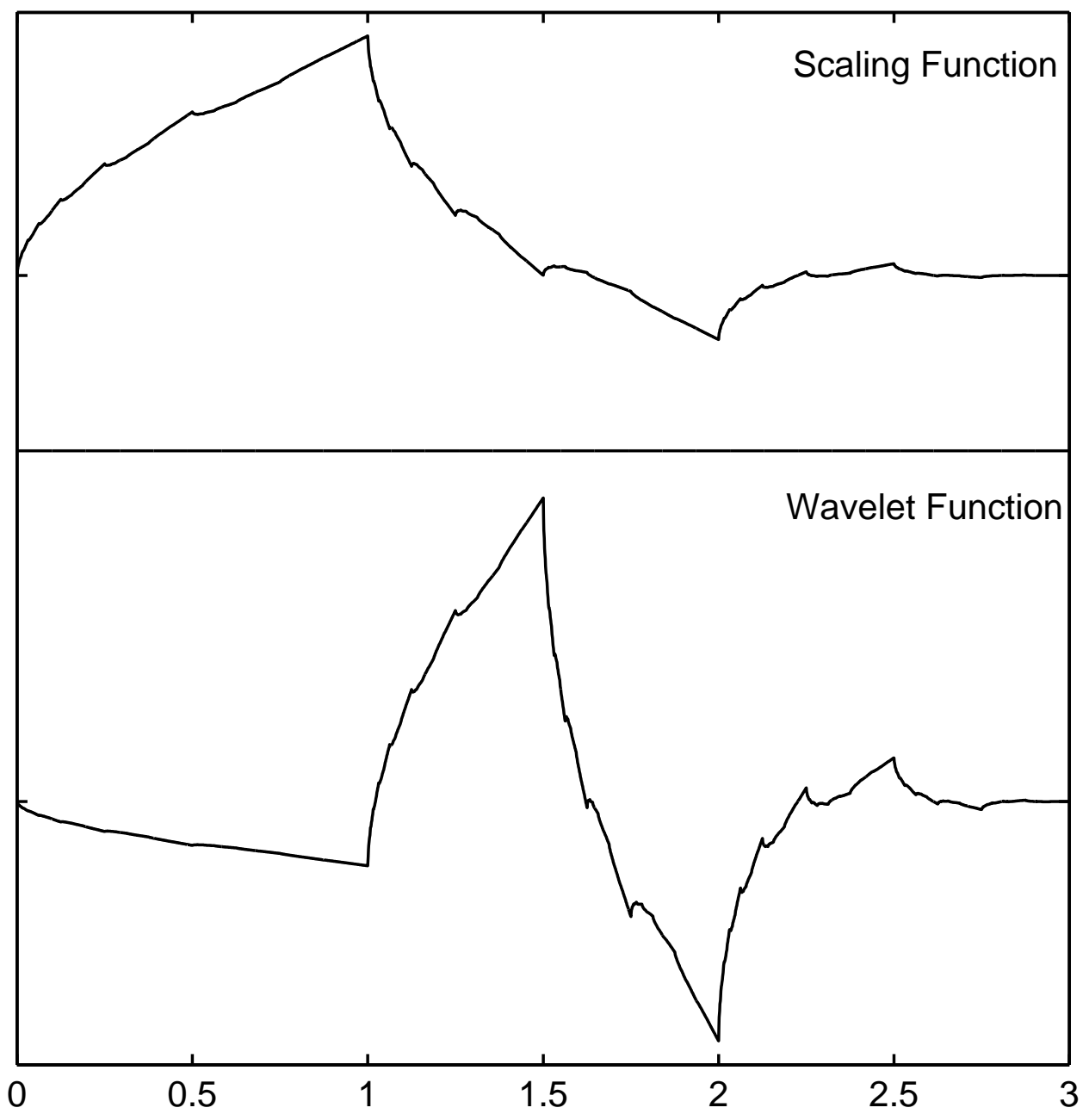


Figure 2: Daubechies $K=3$ Scaling function and mother wavelet function

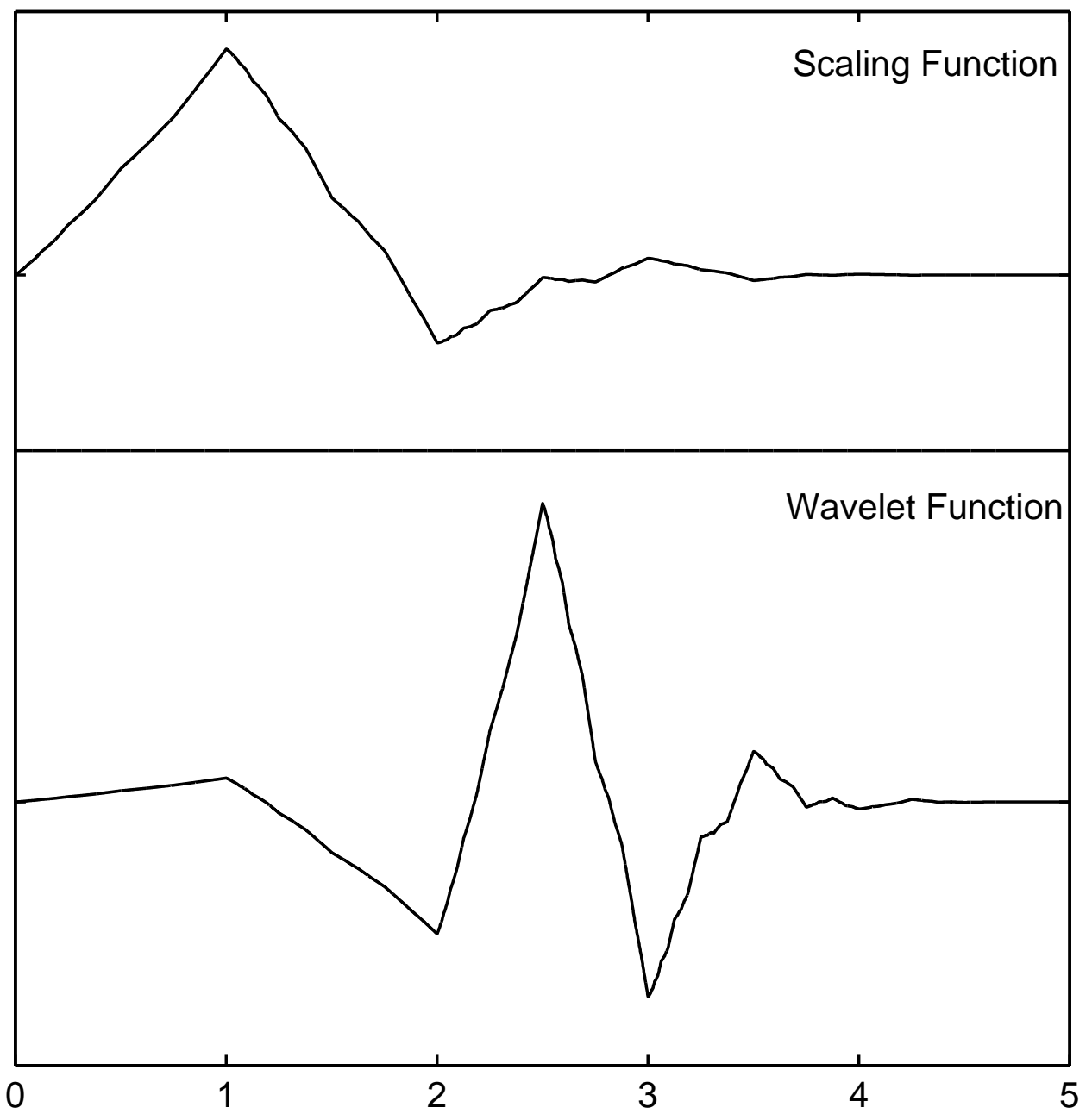


Figure 3: Distribution of wavelet coefficients
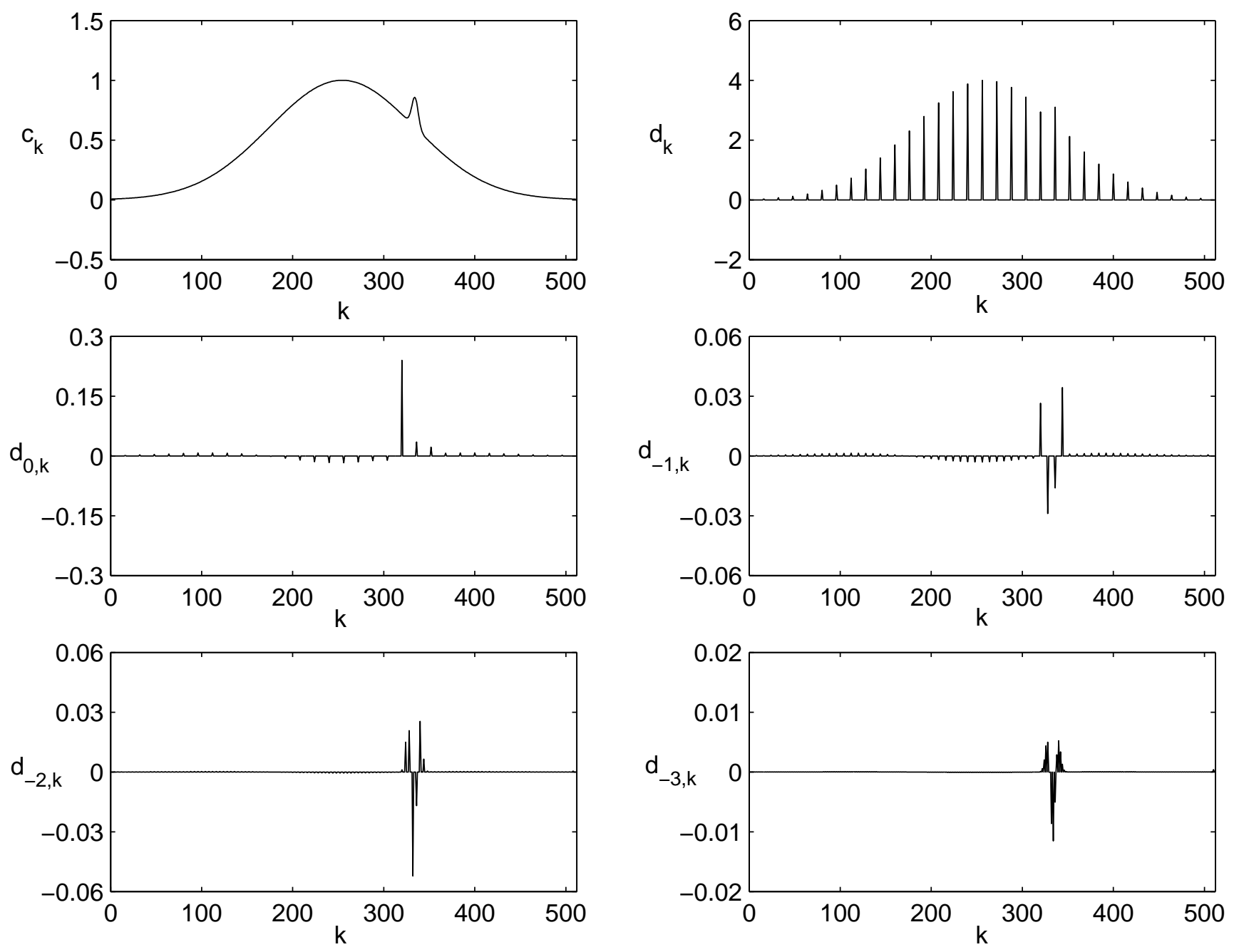
Figure 4: Transformed K-Matrix - E $=80 \mathrm{MeV}$ - expansion in wavelet basis

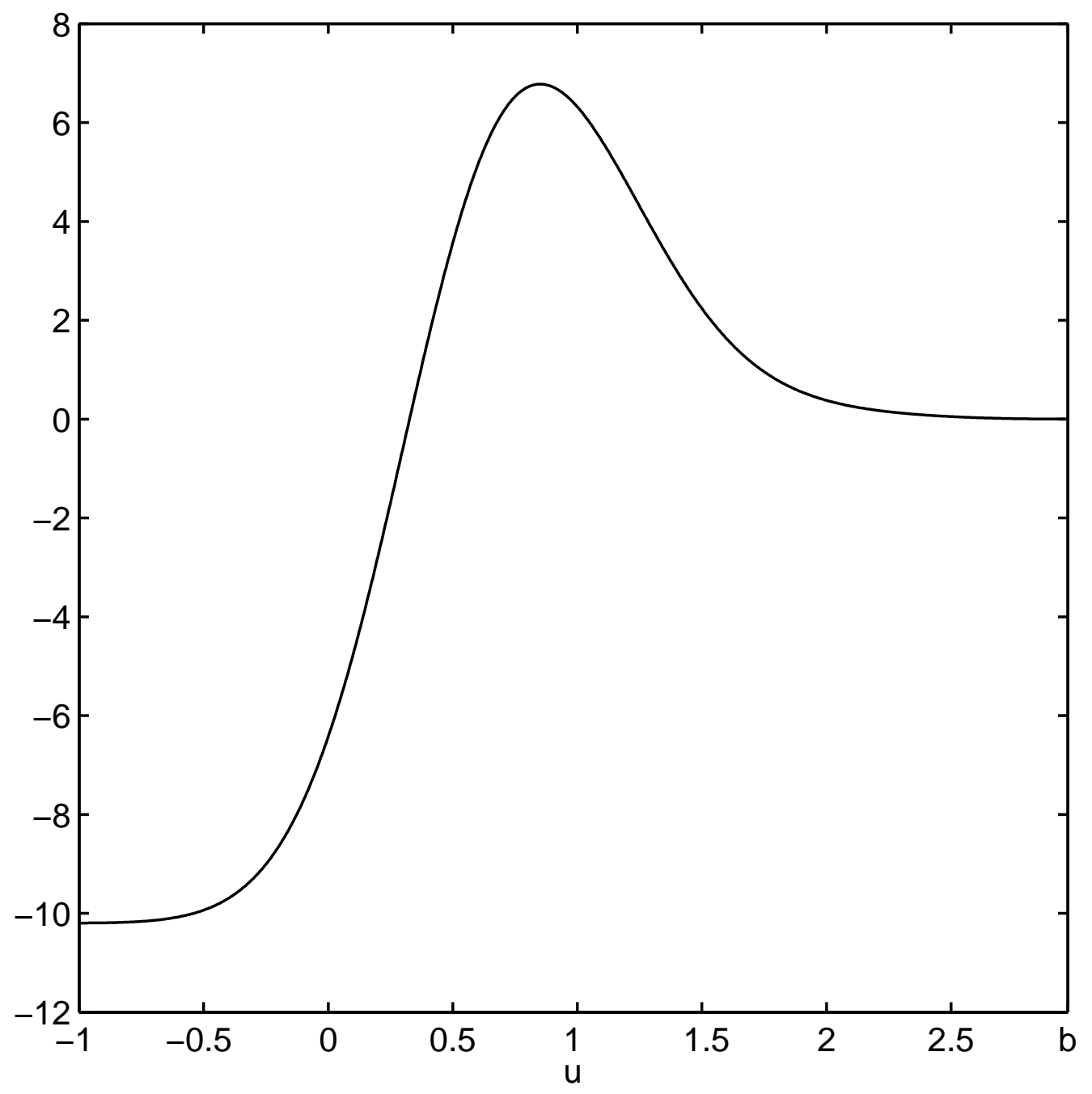


Figure 5: K-Matrix - E $=80 \mathrm{MeV}$ - refined solution

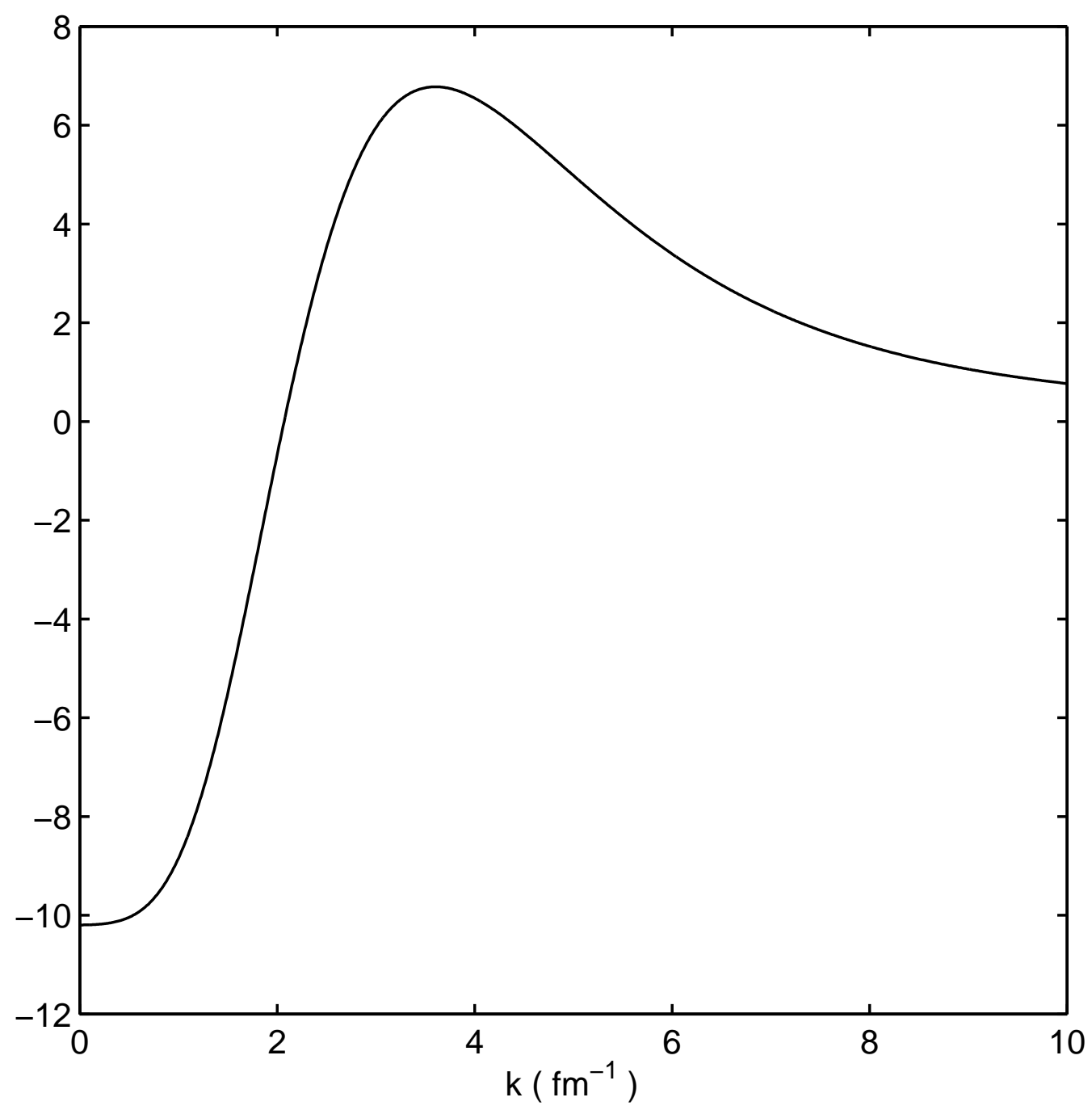


Figure 6: Sparse Kernel for $K=3, \epsilon=10^{-6}$

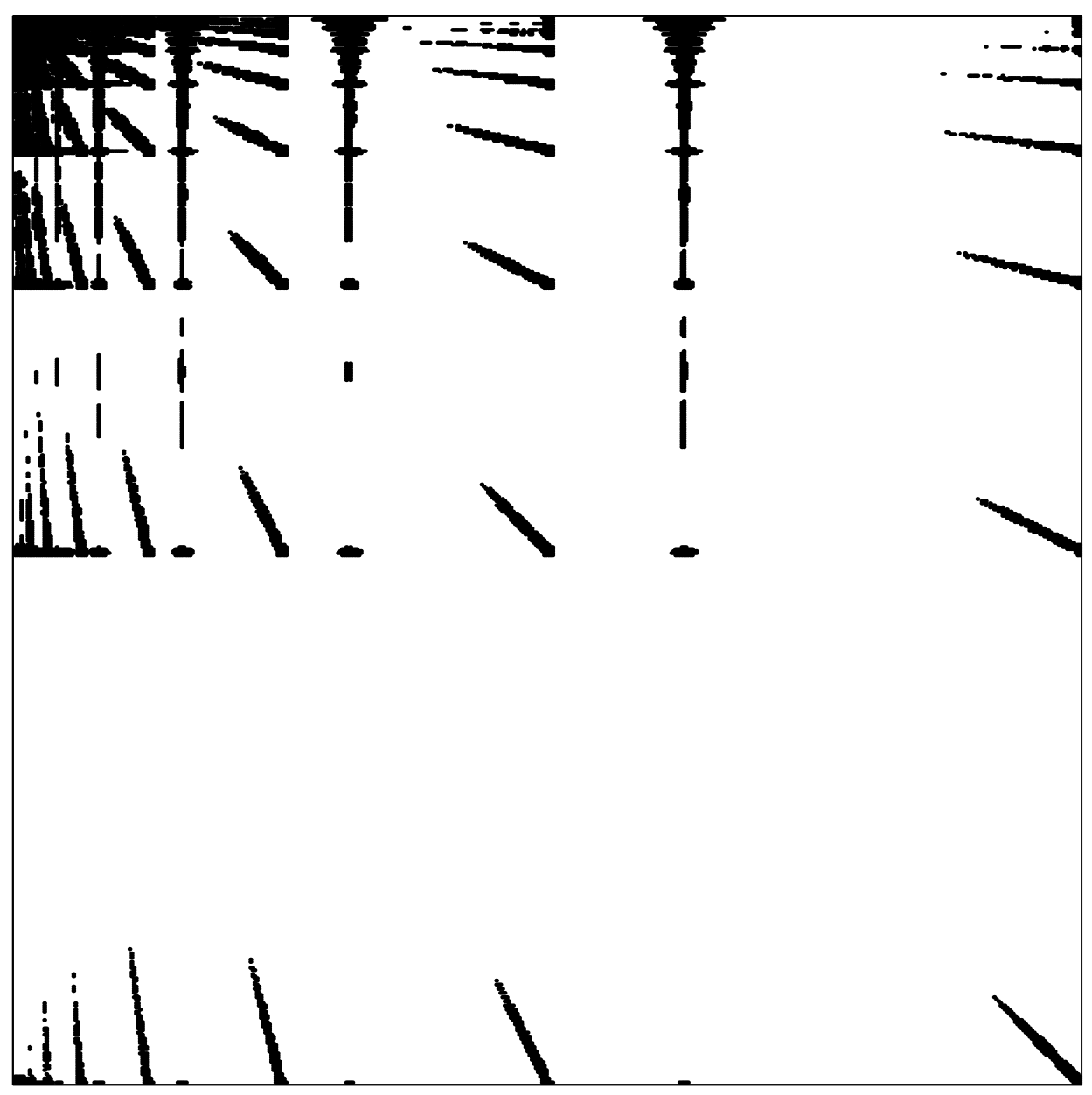

